\title{
For stable Weber B ankle fractures, is three weeks of casting or orthosis application non-inferior to six weeks of cast immobilization?
}

\author{
Riley Golby, MD*; Andrew Kestler $\mathbb{D}, \mathrm{MD}, \mathrm{MScPH}^{*}+\ddagger$
}

\begin{abstract}
link: https://www.bmj.com/content/364/ bmj.k5432.long

Full citation: Kortekangas T, Haapasalo H, Flinkkilä T, et al. Three-week versus six-week immobilization for stable Weber B type ankle fractures: randomized, multicenter, non-inferiority clinical trial. BMJ 2019;364:k5432.
\end{abstract}

Article type: Randomized non-inferiority trial

Ratings: Methods - 4/5 Usefulness - $3 / 5$

\section{INTRODUCTION}

\section{Background}

Weber B fractures are distal fibular fractures that occur at the level of the ankle syndesmosis, with variable stability depending on ligament integrity at the mortise. Increasing evidence suggests that early biomechanical stress in stable Weber B fractures promotes healing, whereas conventional management has been 6 weeks of cast immobilization. Though there are known associated harms with longer-term casting, it remains unclear whether patient functional outcomes are equivalent with shorter periods of immobilization.

\section{Objectives}

This study examines functional outcomes in stable Weber B fractures by comparing 3-week to 6-week cast immobilizations, and by comparing 3-week removable orthosis to 6-week cast immobilization.

\section{METHODS}

\section{Design}

Randomized pragmatic non-inferiority trial

\section{Setting}

Two major trauma centres in Finland from December 22, 2012, to June 6, 2016

\section{Subjects}

Patients ages 16 years and older presenting with an isolated Weber B type fibular fracture, deemed stable through radiographs and fluoroscopy guided external rotation testing

\section{Intervention}

Random assignment to 6-week cast immobilization, 3-week cast immobilization, or 3-week simple orthosis (removable stirrup splint)

\section{Outcomes}

The primary outcome was assessing for non-inferiority in the Olerud-Molander Ankle Score (OMAS) between treatment arms at 12 months (this is a validated tool on an ordinal scale of 0 to 100 , used to assess functional outcomes in ankle fractures, with higher scores indicating

From the ${ }^{*}$ Department of Emergency Medicine, University of British Columbia, Vancouver, BC; ${ }^{\dagger}$ St. Paul's Hospital, Vancouver, BC; and the ${ }^{\ddagger}$ Centre for Health Evaluation and Outcome Sciences, Vancouver, BC.

Correspondence to: Dr. Riley Golby, Royal Columbian Hospital 330 E Columbia St, New Westminster, BC V3L 3W7; Email: riley.golby@alumni. ubc.ca

(c) Canadian Association of Emergency Physicians 2020

CJEM 2020;22(4):468-470

DOI 10.1017/cem.2020.36 
better function). Secondary outcomes assessed ankle function with alternative scoring systems, pain, quality of life, ankle range of motion, radiographic outcome, and rates of adverse events.

\section{MAIN RESULTS}

Patients totalling 247 were enrolled and randomized, with 212 completing the study. The primary outcome of mean OMAS scores between treatment arms at 52 weeks had a predefined inferiority margin of -8.8 points. The results revealed a difference of 3.6 points $(95 \%$ confidence interval $[\mathrm{CI}]:-1.9$ to $9.1, p=0.20)$ for 3 - versus 6 -week cast immobilization, and 1.7 points (95\% CI: -4.0 to $7.3, p=0.56$ ) for 3-week orthosis versus 6-week cast immobilization. Both differences had CIs crossing zero and neither crossed the predefined threshold of -8.8, indicating no significant difference between group outcomes. There were differences in two secondary outcomes: Ankle plantar flexion was statistically significantly improved by 3.0 degrees for 3 -week orthosis compared to 6-week cast (95\% CI: 0.2 to $5.8, p=0.04$ ), and borderline significance for incidence of deep vein thrombosis with a percentage difference of -6.0 favored 3 -week orthosis compared to 6-week casting (95\% CI: -13.2 to $-0.3, p=0.06)$. There were no differences in pain, quality of life, or radiographic outcome between treatment arms.

\section{APPRAISAL}

\section{Strengths}

- Clear, focused research question

- Appropriate randomization and allocation

- Aside from the interventions, groups treated in the same way throughout the trial

- Use of validated patient-centred functional measures as the primary outcome

- Outcome assessors blinded to treatment allocation when possible

- Follow-up sufficiently long and complete

- Assessment of adverse effects considered in addition to other metrics

- Consistent results between intention to treat and per protocol analysis

\section{Limitations}

- Patient and clinician blinding was not possible due to the nature of the intervention.

- Creation of fitted orthosis is not currently available in most North American emergency department (ED) settings.

- No cost-effectiveness analysis is available of orthosis creation versus casting.

- It is unclear whether groups were similar at baseline given a lack of health information documented.

- The primary outcome assessment at intervals earlier than 12 months may have provided useful functional data to assist patient decision-making, especially if one strategy reached the maximal OMAS score significantly earlier over another.

- Continuous outcome measures like OMAS can be difficult to interpret because a statistically significant change may not necessarily be clinically significant. Ideally, the investigators would have provided and justified an OMAS score that they considered acceptable, which would have permitted an easier comparison within and between experimental groups.

- Results showed poor external validity in large part due to patient selection via in-ED orthopedic fluoroscopic testing to assess for fracture stability; this is not a viable option in most North American ED settings.

\section{CONTEXT}

This study used external rotation testing by having in-ED orthopedists stressing the mortise externally under dynamic fluoroscopy to assess joint stability. Alternatively, clinicians have used less intensive stability assessments, using composite factors like medial tenderness, swelling, and ecchymosis or static radiographic mortise displacement. Historical treatment for stable Weber B fractures has included 6 weeks of cast immobilization, which produces high rates of bony union. While this remains a safe option for fracture healing, associated negative consequences include patient inconvenience, stiffness, skin damage, and deep vein thrombosis. ${ }^{1}$ Orthopedists have challenged this dogma, allowing patients to select cast, brace, or weight-bearing as tolerated options for these injuries. ${ }^{2}$ Justification for liberal strategies in stable ankle injuries is that early weight-bearing will generate mechanical stimulus that promotes healing, ${ }^{3}$ possibly with fewer harms than 
immobilization. The present study is the first high-quality randomized trial to compare these strategies.

\section{BOTTOM LINE}

The management of Weber B ankle fractures remains dependent on fracture stability. Noninferiority trials are designed to show that an alternative treatment (shorter cast length or orthosis, in this case) is not worse than the control (6-week cast) by a predetermined margin (OMAS score difference of 8.8 points, in this case). The study provides evidence that managing stable Weber $B$ fractures with an orthosis is non-inferior to traditional methods and duration of immobilization. Unfortunately, determination of fracture stability is a major barrier to widespread implementation within the ED. A conservative approach of temporary immobilization in the acute phase until re-assessment by an orthopedic physician remains a safe option for ED providers.

Keywords: Emergency medicine, musculoskeletal, trauma

Competing interests: None declared.

\section{REFERENCES}

1. Lin CW, Donkers NA, Refshauge KM, et al. Rehabilitation for ankle fractures in adults. Cochrane Datab Syst Rev 2012;11: CD005595.

2. Goodship AE, Kenwright J. The influence of induced micromovement upon the healing of experimental tibial fractures. 7 Bone foint Surg Br 1985;67:650-5.

3. Hente R, Füchtmeier B, Schlegel U, Ernstberger A, Perren SM. The influence of cyclic compression and distraction on the healing of experimental tibial fractures. 7 Orthop Res 2004;22:709-15. 